\title{
Social alienation caused by precocious puberty and hypothalamic obesity in patients with suprasellar arachnoid cysts - Case studies and literature review of a rare but significant disorder
}

\author{
Agata Halabuda*, Anita Franczak-Young, Lukasz Klasa, Stanislaw Kwiatkowski and Lukasz Wyrobek \\ Polish - American Institute of Pediatrics/Jagiellonian University Kraków, Poland
}

\begin{abstract}
Suprasellar arachnoid cysts (SAC), due to their location, are a separate group, regarding clinical symptoms and therapeutic approach, compared to AC in other locations. For this reason they are more similar to other benign suprasellar lesions. SACs are most often diagnosed in children. Symptoms of SAC depend on the age in which they occurred. The treatment needs the engagement of consultant doctors from many fields: neurosurgery, neurology, ophthalmology, endocrinology and radiology and should be also supported by psychologists.
\end{abstract}

Abbreviations: AC: arachnoid cyst; SAC: suprasellar arachnoid
cyst; BHDS: bobble-head doll syndrome; VC: ventriculocystostomy;
VCC: ventriculocystocisternostomy; SH: Shunt; VE: Virtual Endoscopy.

\section{Introduction}

Arachnoid cysts are benign congenital lesions, most often detected accidentally. They are the most frequent intracranial irregularities and constitute of $1-13 \%$ all detected intracranial masses or $2 \%$ of irregularities accidentally detected during the brain imaging due to convulsions.

\section{The most frequent locations are:}

medial cranial fossa, $50-60 \%$

posterior cranial fossa, $10 \%$,

suprasellar region, $2-12 \%$

other locations (intraventricular cysts, cysts of convexity, cysts of quadrigeminal plate), 10\% [1-11]. The production of cerebrospinal fluid (CSF) with simultaneous opening of the foramen of Magendie occurs from $15^{\text {th }}$ week of foetal life. Also, at this moment CSF starts to flow outside the lumen of the ventricular system. Most probably, ACs form in this period. According to a previous theory, the formation of ACs was related to dissection or diverticulum of the arachnoid mater. The most recent works prove that the meninges of the frontal and temporal lobes in a foetus do not join at the moment of formation of the Sylvian fissure and they stay separate, forming a duplicated arachnoid mater $[4,8]$.

This phenomenon might be induced by a local intrauterine condition, e.g. inflammatory process. This mechanism is mainly attributed to the formation of cysts of Sylvian fissure and cysts of brain convexity, however, in the process of formation of a suprasellar cyst a decisive role is played by a separation of a recess in the Liliequist membrane in such a way that the cyst wall is constituted by fully formed arachnoid mater. There is no unambiguous answer as to why some arachnoid cysts enlarge. The most probable theory explaining this is the valve mechanism. Due to the pumping of CSF from the subarachnoid space to the AC, the volume of the AC increases. Caemaert et al. first described a fissure observed during endoscopy at the site of penetration of the basilar artery through the prepontine arachnoid membrane. Uni-directional valve mechanism allows the fluid to enter the cyst, but outflow is impossible - this, in consequence, leads to the enlargement of the cyst. The function of the valve is synchronized with arterial pulsation [2].

Miyajima et al. proposed a new classification of SAC: type I noncommunicating intra-arachnoid cyst of the diencephalic membrane of Liliequist. For this type, the basilar bifurcation is outside the cyst and it is pushed towards the back, in the direction of the brainstem. Type II: communicating cyst - or a cystic dilation of the interpeduncular cistern. The cyst is located between two layers of Liliequist's membrane and the basilar bifurcation is located inside the cyst $[3,6,12,13]$.

SACs, due to their location, are a specific case of arachnoid cysts and require a separate management; for this reason, they are more similar to a wide group of suprasellar and parasellar masses. Supratentorial tumours of the medial line (sella turcica, optic chiasm) in children constitute $13-16 \%$ of all intracranial neoplasms (including: SAC 2\%).

${ }^{\star}$ Correspondence to: Agata Halabuda, Polish - American Institute of Pediatrics / Jagiellonian University Kraków, Poland, E-mail: agatahalabuda@gazeta.pl

Key words: Arachnoid cyst, suprasellar arachnoid cyst, bobble-head doll syndrome, ventriculocystostomy, ventriculocystocisternostomy, shunt, virtual endoscopy

Received: November 18, 2019; Accepted: December 03, 2019; Published: December 06, 2019 
Halabuda A (2019) Social alienation caused by precocious puberty and hypothalamic obesity in patients with suprasellar arachnoid cysts - Case studies and literature review of a rare but significant disorder

Types and incidence (\%) of individual supratentorial tumours of cerebral hemispheres, medial line and infratentorial tumours in children are shown in Figure 1 [14]. The classification includes only the lesions that cause clinical symptoms and require treatment. It does not include incidentaloma lesions, which, due to progresses in imaging techniques, appear surprisingly often in MR scans, but usually do not constitute a clinical problem. They are detected in $8-10 \%$ of cases in healthy subjects.

Autopsy studies have shown that incidentaloma can happen in individuals without a known pituitary disease in $1.5-27 \%$ cases.

Vision disorder and neurologic disorder occur in $25-85 \%$ of patients with SAC, while various endocrine disorders, including precocious puberty, occasionally with fulminant course (pubertas fulminans), occur in $10-40 \%$ of children with SAC [10,14-19].

Most SAC become symptomatic in early childhood. $60-90 \%$ of all symptomatic SACs described in literature are in children.

Macrocrania and psychomotor retardation are the most common symptoms in infants, while psychomotor retardation, seizures and precocious puberty occur in older children with SAC [2,3,15,16,20,21].

Suprasellar lesions are mostly benign, but due to their location they are challenging for interdisciplinary treatment - neurosurgical, neurological, endocrine, ophthalmic and psychological.

In our work, we analyzed patients with symptomatic suprasellar arachnoid cysts retrospectively who were treated at the University Children's Hospital of Krakow. They presented an age-dependent, wide spectrum of clinical symptoms, i.e.: endocrine disorders (centraltype precocious puberty), hypothalamic obesity, hydrocephaly, vision disorders, emotional disorders and psychomotor retardation.

Bobble-head doll syndrome (BHDS) is a relatively rare symptom. BHDS did not occur in any of the patients described by us $[2,16,8,20]$. The analysis of our patients included family history, prenatal examination, delivery and the perinatal period, the age of the patient at the moment of lesion detection, clinical symptoms as well as methods and results of treatment.

\section{Radiologic diagnosis}

An important component of diagnosis and therapy is correct interpretation of imaging studies, as suprasellar cysts can mimic a dilated third ventricle and be misdiagnosed as aqueduct stenosis, which changes the approach in an essential way. Wang et al. presented three characteristic features differentiating these two units. For SAC, the optic chiasm is vertically displaced, mammillary bodies are displaced upwards and the pons is displaced ventrally. These features differentiate SAC from hydrocephaly caused by aqueduct stenosis, where mammillary bodies and the floor of the third ventricle are slightly inferiorly displaced $[3,11]$. It should be remembered that if the cyst enlarges and closes the third ventricle, blocking the aqueduct, this finally also leads to hydrocephaly.

\section{Clinical symptoms}

Precocious puberty: is defined as physical and hormonal changes that occur in girls before the age of 7-8 years (for Afroamericans before the age of 6 years) and in boys before 9 years of age [22-24]. Central precocious puberty (associated with a damage of hypothalamicpituitary axis) is caused by a premature activation of neurons present in the hypothalamus, releasing gonadoliberin. Despite a significant improvement in diagnostics, including also imaging tests, it is impossible to determine the cause of precocious puberty in $70-90 \%$ of girls and $10-50 \%$ of boys - these cases are called idiopathic central precocious puberty. The remaining cases are most often caused by proliferative processes/suprasellar lesions. Less frequent causes of central precocious puberty include iatrogenic lesions, genetic disorders (type 1 neurofibromatosis, Williams-Beuren syndrome), exposition to pesticides. Also cases of precocious puberty were reported in children in whom intracranial bleeding and/or a temporary increase of intracranial pressure was observed during the neonatal period. Apart from iatrogenic lesions caused by neurosurgical treatment of suprasellar tumours, also other iatrogenic causes should be noted, such as preventive irradiation of the brain with low and medium radiation doses up to $24 \mathrm{~Gy}$, e.g. for acute lymphoblastic leukemia. In contrast to high radiation doses (above 40 Gy), which inhibit the hypothalamic-pituitary axis, low and medium doses might cause precocious puberty. The mechanism of this phenomenon is not entirely known, probably there occurs an imbalance between the factors stimulating and inhibiting the function of pulse generator for endogenous gonadoliberin [22-24].

Hypothalamic obesity: The control of food intake is a very complex process, not completely elucidated. Participating in this process are many antagonistic mechanisms, which regulate it at the central level as well as at the peripheral level. The main site responsible for the integration of different signals is the hypothalamus and the arcuate nucleus controlled by hypothalamic AMP-activated kinase, as well as the prefrontal cortex, the amygdala and the brainstem. At the peripheral level this process is regulated by hormones and peptides, such as adiponectin, leptin, ghrelin, insulin and glucagon $[25,26]$.

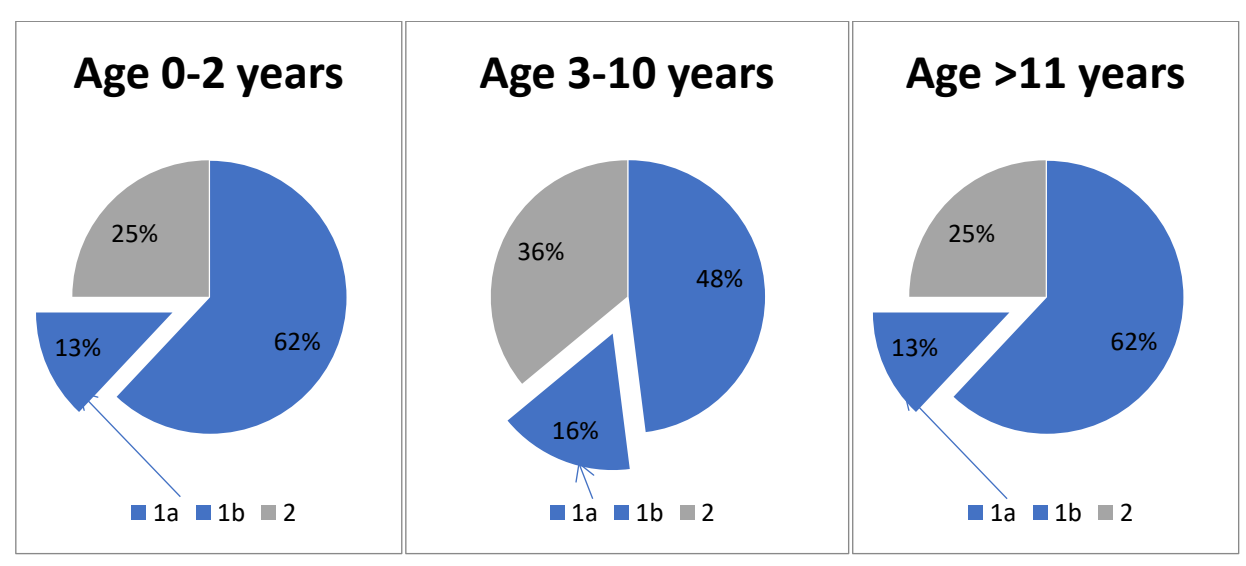

Figure 1. 1) Supratentorial tumours (a. cerebral hemispheres, b. medial line), 2) Infratentorial tumours 
Halabuda A (2019) Social alienation caused by precocious puberty and hypothalamic obesity in patients with suprasellar arachnoid cysts - Case studies and literature review of a rare but significant disorder

In cases of supra- and parasellar lesions, obesity is associated with hypothalamus damage, nevertheless, this disorder can occur also in patients who were not subject to too invasive procedures and/ or patients whose treatment includes complete hormonal regulation, which undoubtedly reinforces the theory about the complexity of the process of food intake control.

There is discussion as to whether hyperinsulinemia is linked to hypothalamus damage or is secondary to hyperphagia. Other authors suggest that the increased level of leptin is a more important obesity factor than increased insulin secretion. An additional risk factor for obesity development in individuals with tumours of the suprasellar region is the lack of physical activity, which could be linked to emotional disorders and/or mental retardation, which is why psychological support is a necessary component of the management. Interesting data on obesity is provided by a study using $\mathrm{fMR}$, where authors demonstrate that in this complex process the reward system (nucleus accumbens) is also altered. Patients from the control group and patients after surgery for craniopharyngioma were tested using $\mathrm{PMR}$, with activity evaluation within the nucleus accumbens before and after a meal. Post-prandial suppression of neural activity caused by visual food cues was clearly blunted in individuals with craniopharyngioma, thereby these patients were still hungry, unlike the patients in the control group [27].

Visual disorders: most often they are caused by hypoplasia of the optic nerve due to its' compression by a cyst or other type of suprasellar lesion. Visual disorders might also occur as iatrogenic lesion after surgical treatment where the optic chiasm or optic nerve was damaged $[17,18]$.

Bobble-head doll syndrome: About $10 \%$ of children with suprasellar cyst have this symptom. BHDS most often affects children below 10 years of age and is characterized with backward head movements that might also include the neck, trunk and upper limbs. This symptom disappears during sleep and during focusing on a task, while it intensifies when walking or during excitation. The pathomechanism of this phenomenon is not entirely understood. Rhythmic head movements could be caused by the pressure of pulsating cerebrospinal fluid, which spreads to adjacent structures, including the thalamus [28].

Emotional disorders and mental retardation: In some cases SACs are concomitant with such disorders as obsessive-compulsive behavior, aggression, stereotypies and mental retardation. These symptoms are usually secondary to endocrine disorders and hydrocephaly caused by SAC. Optimal cognitive functioning requires correct concentrations of estrogen, testosterone and corticosteroids. Deviations of concentrations of the above-mentioned hormones from the normal range cause, among other things, disorders of attention, verbal memory and spatial skills. Re-establishing the hormonal balance usually gives a significant improvement, that is why proper diagnosis as early as possible, is so important, especially in the case of the still-developing brain of a child.

It should be emphasized that the somatic symptoms resulting from damage to the pituitary / hypothalamus areas, such as obesity or precocious puberty, directly affect social and family functioning. Increasing obesity causes gradual withdrawal from activities, e.g. sports, but also other types of activities that require social interaction. This results a lower self-estimation of the childs' efficacy / effectiveness and may cause a reduction in academic performance, which is not due to impairment of the childs' intellectual abilities. Precocious puberty brings with it the risk of premature sexual behavior, early sexual initiation and the possibility of pregnancy at an early age are a significant burden for young patients. They are not prepared to cope with such difficult challenges on their own due to emotional development that "cannot keep up" with their sexual development. It also causes difficulties in functioning within their peer group and a lack of acceptance from their milieu. The consequences can include aggression or an increased risk of addiction to alcohol and psychoactive substances used to relieve tension. All of these factors can, in the long term, result in functioning below the patient's abilities, the emergence of social pathology and a decrease in socio-economic status.

Patients with precocious puberty caused by a suprasellar cyst or tumour should receive psychological help from the moment they are diagnosed. There is a need for assessment of intellectual functioning, a neuropsychological diagnosis that would detect possible deficits in cognitive function (e.g. attention deficit disorder) and an assessment of emotional functioning. All this would specify the types of psychological support / therapy that the child should then receive. Systemic psychological help, which would include the whole family, would be ideal. Already at an early stage of the disease, the entire family which is involved in the treatment process should be able to benefit from psychoeducation about the nature of the disorder and its possible consequences. During such education, family members also have the opportunity to work through their difficulties in dealing with individual aspects of the disease, which for each of them can be completely different. Often, against the background of these differences, families of chronically ill people create high tensions and conflicts that could be avoided by early intervention. In the event of a deterioration in the functioning of the family, all members should be provided with psychotherapy. Also, the child himself, in addition to neurosurgical and endocrine control, should have a dedicated psychotherapist. Currently, unfortunately, there is no such holistic approach to the treatment of children suffering from SAC.

Treatment methods: In the case of suprasellar cysts, the indications for surgical treatment are still a subject of discussion. Most authors agree that small, asymptomatic cysts do not require surgical treatment. Currently, the following conditions are considered as absolute indications for surgical treatment: hydrocephaly, symptoms of increased intracranial pressure and progression of the cyst's dimensions in subsequent imaging tests. For other cases, there are supporters of conservative treatment as well as those of surgical treatment $[3,8,13,20,21]$.

Some authors indicate that for large, asymptomatic suprasellar cysts which are not growing, conservative treatment in the form of strict clinical and radiological monitoring is adequate. An argument supporting this concept includes the results of autopsy examinations performed in patients deceased for other reasons than increased intracranial pressure, who are often found to have arachnoid cysts. An additional argument refers to individual cases, described in literature, where SAC spontaneously disappeared during the observation period $[7,15]$.

On the other hand, some authors report that surgical treatment of symptomatic cysts, especially if the symptoms of precocious puberty are already present, is performed too late. Therefore, the supporters of surgical treatment of large asymptomatic AC justify their point of view with a high risk of later potential complications. Thus, when considering alternative methods, it is important to remember that the occurrence of endocrine disorders in patients with SAC is irreversible in most cases. However, even in such cases, the interventional treatment should be performed before pharmacotherapy, in order to prevent further hormonal dysfunction. 
Halabuda A (2019) Social alienation caused by precocious puberty and hypothalamic obesity in patients with suprasellar arachnoid cysts - Case studies and literature review of a rare but significant disorder

Interventional treatment techniques are: 1) microsurgery 2) endoscopy and 3) shunt. The first two methods aim to create connections (fenestration) between the cyst lumen and arachnoid cisterns and/or ventricular system. The third method is used as a supportive treatment for the first two or when they prove to be ineffective [2,3,29,30-32].

The microsurgical technique is the most invasive of the above mentioned methods, however, it is highly effective, due to the ability to create a wide opening. An advantage of this technique is a wide access to the operative field, the possibility for immediate intervention in the case of vascular complications; it is also easier to locate and destroy the valve mechanism in SAC. The fenestration procedure itself, with a wide opening of 1-1.5 cm, preventing re-occlusion, is also safer and easier.

The complications include formation of intracerebral and subdural hematomas, cranial nerve palsies and aseptic meningitis. However, the most important shortcoming is a high mortality rate, in the range of $1-10 \%[2,16]$. Nevertheless, most authors claim that currently the best method of proceeding is the use of endoscopic techniques. These techniques combine the high effectiveness, attributed to microsurgical procedures, with low invasiveness, specific for the implantation of valvular systems [33]. Approaches for accessing the suprasellar region in endoscopic techniques are: transfrontal/transcallosal and endonasal/ transsphenoidal. The transfrontal approach causes larger damage to the cortex and hypothalamus, which leads to a risk of later neurologic and endocrine complications. The endonasal approach seems to be safer due to a lower risk of damaging the brain structures, but it requires a highly experienced operator, and this technique is not indicated for every case. In recent years, the so called extended endoscopic approach, EES, was introduced into clinical practice, allowing access to almost the complete cranial floor, including the suprasellar area, through the nasal cavity. This method, although it transforms a tumour of the base of skull to a tumour located superficially, is not a minimally invasive procedure. This is because it covers a wide opening of the base of the skull, which requires the removal of many anatomical structures [12,30,32,34].

The limitation of the endoscopic method is a small sella tunica. Technical problems may also arise from a fast collapse of the suprasellar cistern to a residual cavity as well as venous bleeding or infiltration of CSF inside the sella after the evacuation of the cyst. When this method is to be used in the treatment of SAC, the problem is whether there is communication between the SAC and subarachnoid space (the valve mechanism mentioned above), due to the limited possibility of evaluating the operative field. In such cases, an empirical method could be used, i.e. observation of refilling of the cavity of the sella turcica after having it emptied, but when the communication path is very small, this method might be ineffective. Endoscopic procedures are the most useful only for lesions with a large and predominant suprasellar development $[2,16]$. Recently, some reports have appeared concerning another important complication of endoscopic procedures, related to too aggressive irrigation of the operative field, which can lead to a critical increase in intracranial pressure and sudden cardiac arrest [35]. The fenestration procedure can be performed creating a single connection between the cyst lumen and the ventricular system, VC (ventriculocystostomy), or creating two connections with the ventricular system and arachnoid cisterns, VCC (ventriculocystocisternostomy). Many authors consider VCC to be the more effective method, because the creation of two connections reduces the risk of re-occlusion. However, it is not always possible to use this method, due to anatomical considerations [9].

Virtual endoscopy (VE) could be helpful in choosing a surgical method and procedure planning $[5,35]$.
Cystoperitoneal/ventriculoperitoneal shunt was previously used as the first-line treatment. After developing of techniques including creating connections between the cyst and the arachnoid cisterns and/ or ventricular system, it is used only in the case of failure of the above methods or as a supplementary technique, e.g. for hydrocephaly not resolving after the current first-line treatment methods. About $60-70 \%$ of patients with a suprasellar arachnoid cyst additionally require the implantation of a shunt system $[8,29]$. Even though this procedure has the lowest risk of intra-operational complications, the lethal complications rate oscillates around zero, in a longer perspective the prognoses are clearly less favorable than in the case of fenestration. First of all, so-called shunt dependency might occur, or an acquired impairment of absorption capacity of arachnoid granulation as a result of using an external drainage system. After a year, only $60 \%$ of shunts function effectively, and after 5 years - about $40-50 \%$; this means that about $50-60 \%$ of patients require a re-intervention within 5 years of the shunt implantation. Everyday life also requires a certain regime - the patient should be under the constant supervision of an ophthalmologist and psychologist, as the symptoms of shunt failure are not always acute symptoms of increasing intracranial pressure. Moreover, intensive, long-term physical activity is contraindicated. As stated by Aschoff ,an ideal shunt is no shunt" $[29,36]$.

The literature describes also rarely used, unusual methods of treatment of arachnoid cysts, including stereotactic implantation of P32 isotope into the cyst lumen [37].

\section{Discussion}

Among our patients, the dependence between age and presented symptoms is clearly visible. The first group are the children, between 1 and 16 months of age, who have shown such symptoms as excess increase of head circumference and retarded psychomotor development. The second group consists of older children, between 5 and 10 years of age, who have precocious puberty as a dominating symptom. This symptom was the direct reason for contacting a physician presents aggregated data from all the discussed cases.

One child (patient No. 6) presented a disease course different from the others - in the first year of life a ventriculoperitoneal shunt was implanted for hydrocephaly. Up to 7 years of age no alarming symptoms were observed, the shunt system functioned properly, the patient did not require any surgical interventions. At the age of 7 , the symptoms of precocious puberty occurred, and imaging of the brain showed a SAC that was not present in previous examinations.

In all the children, no pathologic family history or abnormalities during pregnancy, birth and the perinatal period were found. All the children were qualified for surgical treatment - fenestration. In 3 children a craniotomy procedure was performed. Other children had an endoscopic procedure. In two children (No. 5 and No. 8), an occlusion of the communication path between the cyst lumen and the arachnoid cisterns occurred, after 3 and 6 years, respectively.

As a consequence, the arachnoid cyst enlarged, and hydrocephaly, moderate and stationary up to that moment, increased.

Additionally, in imaging studies, periventricular infiltration of CSF into the white matter (intraventricular hypertension) was observed. Reoperation was performed for the children. Patient No.5 had endoscopic VC, patient No.8 underwent a craniotomy with a shunt between the cyst lumen and the basal cisterns. Additionally, this patient had a ventriculoperitoneal shunt installed. In 2 children (patients No.3 and No.4), the hydrocephaly persisted despite the performed treatment. 
Halabuda A (2019) Social alienation caused by precocious puberty and hypothalamic obesity in patients with suprasellar arachnoid cysts - Case studies and literature review of a rare but significant disorder

After a dozen days from the fenestration, a ventriculoperitoneal shunt was additionally installed. 4 children showed the features of psychomotor retardation during long-term observation. These children required rehabilitative treatment and stayed under neurologic, neurosurgical and psychological supervision. 4 children diagnosed with central precocious puberty, confirmed during diagnostics in LH$\mathrm{RH}$ test, remain under endocrinological supervision and were treated with long-acting analogues of GnRH. In 3 children with precocious puberty, = an incorrect body weight was found (one child was obese, two were overweight). One patient with precocious puberty had a body weight appropriate for his age and height (Figures 2-5) show patient No.7 before and after the surgical treatment of SAC.

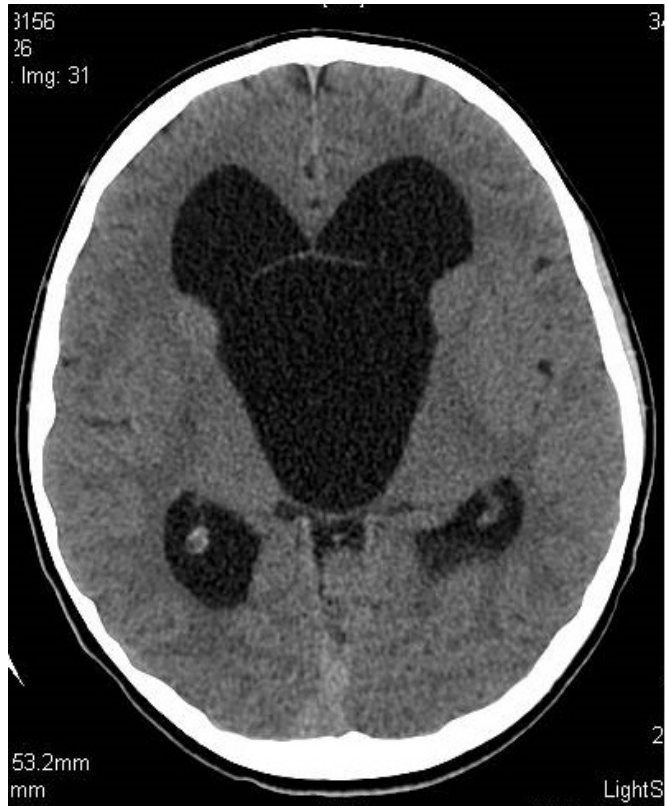

Figure 2. Preoperative axial scan. Non-enhanced CT. Section at the level of Monro foramina. SAC and hydrocephaly. Mickey Mouse syndrome

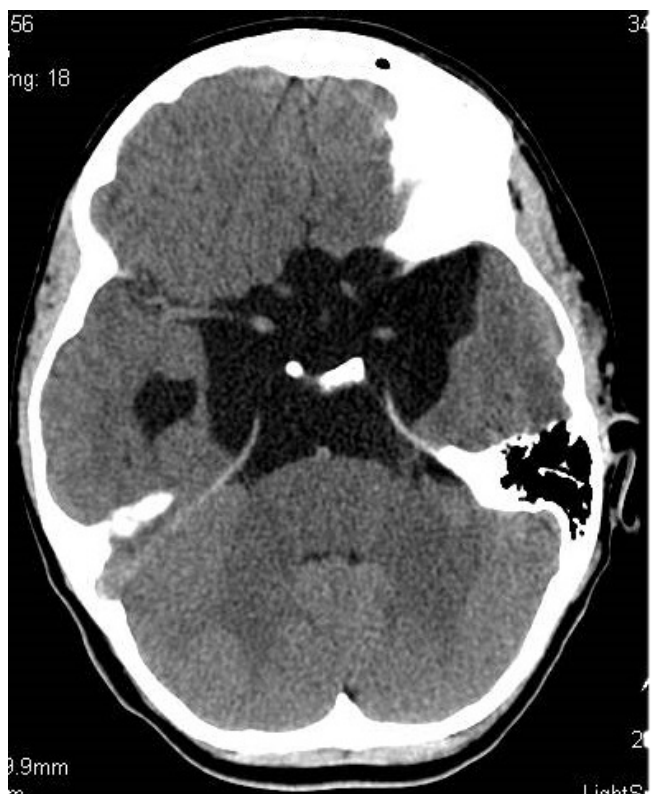

Figure 3. Section at the level of basal cisterns

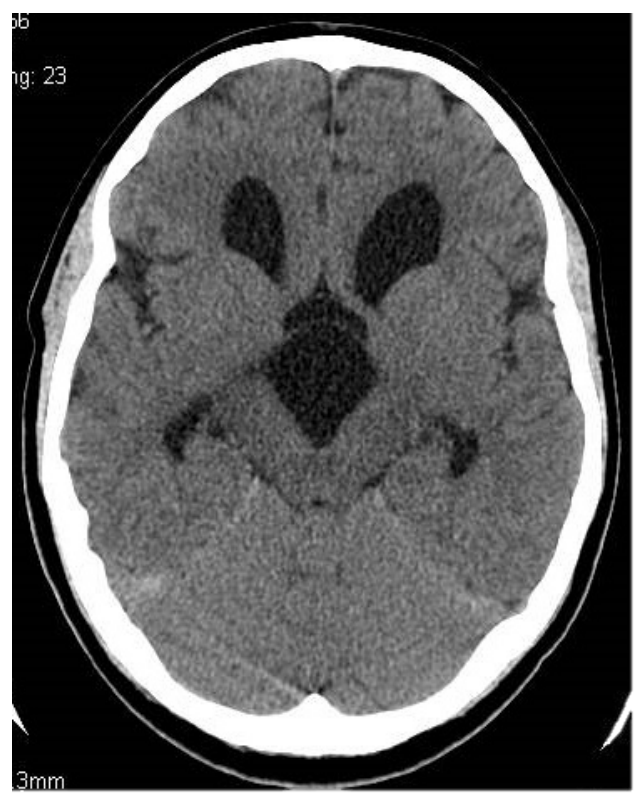

Figure 4. Postoperative axial scan.Non-enhanced CT. Section at the level of Monro foramina

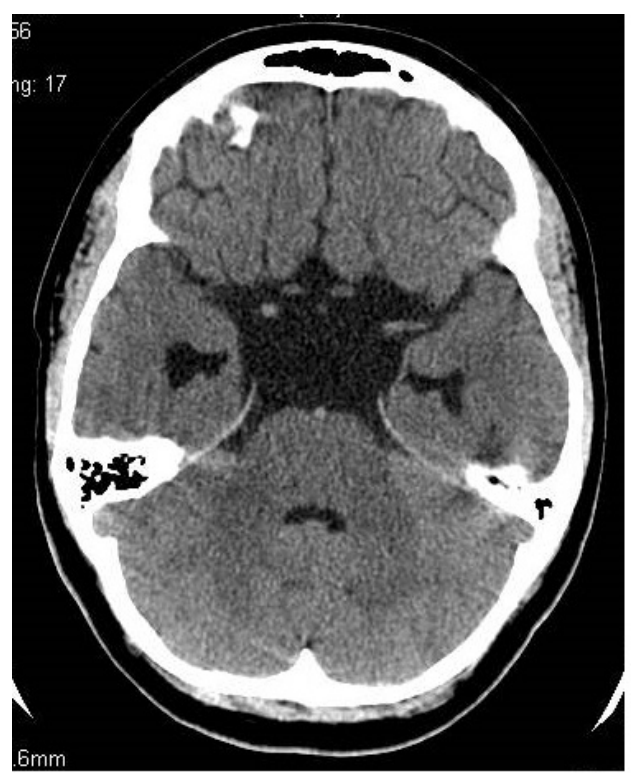

Figure 5. Section at the level of basal cisterns. SAC and hydrocephaly regression. 3 years after operation

\section{Conclusions}

1. Symptoms of SAC are age dependant.

2. The indications for surgical treatment pertain to patients with symptomatic SAC. Currently, the best method of surgical treatment is the endoscopic technique including the formation of two connections: with the arachnoid cisterns and with the ventricular system. As opposed to most patients with AC in other locations, patients with SAC more often require the additional implantation of a shunt system due to persisting hydrocephaly.

3. If precocious puberty occurs in patients with SAC, the symptoms will not resolve after neurosurgical treatment however in spite of this, the treatment is necessary to prevent further hormonal dysfunction. 
Halabuda A (2019) Social alienation caused by precocious puberty and hypothalamic obesity in patients with suprasellar arachnoid cysts - Case studies and literature review of a rare but significant disorder

In such cases, the next stage of treatment is therapy with long-acting analogues of $\mathrm{Gn} \mathrm{RH}$.

4. Patients underwent strict neurosurgical and endocrinological control (including the treatment of obesity). systemic psychological care of the patient and his family is insufficient.

5. The suprasellar lesion as well as complications after neurosurgical surgery may cause varying degrees of damage to the structures of the hypothalamic pituitary region, the consequence of which are symptoms of varying severity: precocious puberty, obesity, psychomotor delay, emotional disorders. Patients with precocious puberty caused by a suprasellar cyst or tumour should receive psychological help from the time they are diagnosed. 6. The family should have access to psychoeducation about the nature of this disease and the possible social consequences. In cases of deterioration in the functioning of the family, all members should be provided with psychotherapy.

\section{Conflicts of interest}

All authors have no affiliations with or involvement in any organization or entity with any financial interest or nonfinancial interest in the subject matter or materials discussed in this manuscript.

\section{Financial support}

This work is not financially supported by any project.

\section{References}

1. Andre A, Zerah M, Roujeau T, Brunelle (2016) Suprasellar arachnoid cysts: Toward a new simple classification based on prognosis and treatment modality. Neurosurg 78 : 370-380.

2. El-Ghandour NMF (2011) Endoscopic treatment of suprasellar arachnoid cyst in children. J Neurosurg Pediatrics 8: 6-14.

3. Gui S, Wang X, Zong X (2011) Suprasellar cyst: Clinical presentation, surgical indication and optimal surgical treatment. BMC Neurology 11: 52 .

4. Jones BV (2005) Cyst and cyst-like lesion. In Donelly LF (ed) Diagnostic imaging pediatrics 1st ed. Amirsys publishing, Inc 7: 86-90.

5. Li Y, Zhao Y, Zhang J (2016) Low-cost interactive image-based virtual endoscopy for the diagnosis and surgical planning of suprasellar arachnoid cyst. World Neurosurgery 88: 76-82.

6. Miyajima M, Arai H, Okuda O (2000) Possible origin of suprasellar arachnoid cyst: neuroimaging and neurosurgical observations in nine cases. J Neurosurg 93: 62-67.

7. Moon K, Lee J, Kim J (2007) Spontaneous disappearance of a suprasellar arachnoid cyst: case report and review of the literaturę. Childs Nerv Syst.

8. Nowoslawska E (2007) Arachnoid cyst In: Zakrzewski K(ed) Hydrocephalus and the other disturbances of cerebro-spinal fluid circulation in children. 1st ed. Wydawnictwo Czelej Sp. Z o.o. Chapter 14, pp: 143-155.

9. Rizk E, Chern JJ, Tagayun C et al (2013) Institutional experience of endoscopic suprasellar arachnoid cyst fenestration. Childs Nerv Syst 29: 3-13.

10. Starzyk J, Kwiatkowski S, Urbanowicz W (2003) Suprasellar arachnoid cyst as a cause of precocious puberty - report of three patients and literature overview. Journal of Pediatric Endocrinology and Metabolism 16: 447-455.

11. Wang JC, Heier L, Souweidane M (2004) Advances in the endoscopic management of suprasellar arachnoid cyst in children. J Neurosurg Pediatrics 100: 418-426.

12. Mortazavi MM, Rizq F, Harmon O (2014) Anatomical variations and neurosurgical significance of Liliequist's membrane. Childs Nerv Syst.
13. Ozek MM, Urgun K (2013) Neuroendoscopic management of suprasellar arachnoid cyst. World Neurosurg 79(2 Suppl): S19.e13-8. [Crossref]

14. Bladowska J, Sokolska V, Czapiga E (2004) Advances in diagnostic imaging of the pituitary and the parasellar region. Adv Clin Exp Med 4: 709-717.

15. Adan L, Bussieres L, Dinand V (2000) Growth, puberty and hypothalamic-pituitary function in children with suprasellar arachnoid cyst. Eur J Pediatr 159: 348-355.

16. Cavallo LM, Prevedello D, Esposito F (2008) The role of the endoscope in the transsphenoidal management of cystic lesion of the sellar region. Neurosurg Rev 31: 55-64. [Crossref]

17. Mohn A, Schoof E, Fahlbusch R (1999) The Endocrine spectrum of arachnoid cyst in childhood. Pediatr Neurosurg 31: 316-321.

18. Mohn A, Fahlbusch R, Dorr HG (1999) Panhypopituitarism associated with diabetes insipidus in a girl with a suprasellar arachnoid cyst. Horm Res 52: 35-38.

19. Okamoto K, Nakasu Y, Sato M (1981) Isosexual precocious puberty associated with multilocular arachnoid cyst at the cranial base. Acta Neurochirurgica 37: 87-93.

20. Pierre-Kahn A, Capelle L, Brauner R (1990) Presentation and management of suprasellar arachnoid cyst. J Neurosurg 73: 355-359.

21. Rabiei K, Hogfeldt MJ, Doria-Medina R (2016) Surdery for intracranial arachnoid cyst in children - a prospective long-term study.Childs Nerv Syst 32: 1257-1263. [Crossref]

22. Krysiak M, Marek B, Okopien B (2008) Central precocious puberty. Polish Journal of Endocrinology 59: 530-540

23. Krysiak M, Szkrobka W, Kowalska B (2014) Precocious puberty in boys. Medical Review 71: 549-557.

24. Manjila S, Vogel T, Chen Y (2014) Hypothalamic hamartoma simulating a suprasellar arachnoid cyst: resolution of precocious puberty following microsurgical lesion resection. J Neurosurg Pediatrics 14: 101-107.

25. Fijalkowski F, Jarzyna R (2010) Role of hypothalamic AMP-activated protein kinase in the control of food intake. Postepy Hig Med Dosw 64: 231-243.

26. Nolte J (2011) Thalamus and capsula interna - communication with cerebral cortex In: Nolte J (ed) The Human Brain. An Introduction to ist Functional Anatomy. 1st ed. Elsevier Urban\& Partner.Chapter 16, PP: 50-75.

27. Roth CL, Aylward E, Liang O (2012) Functional neuroimaging in craniopharyngioma: A useful tool to better understand hypothalamic obesity? Obes Facts 5: 243-253. [Crossref]

28. Hagebeuk E, Kloet A, Grtenhuis (2005) Bobble-head syndrome successfully treated with an endoscopic ventriculocystocisternostomy. J Neurosurg (pediatrics 3) 103: 253 259

29. Kim S, Cho B, Chung Y (2002) Shunt dependency in shunted arachnoid cyst: A reason to avoid shunting. Pediatr Neurosurg 37: 178-185.

30. Lyson T, Sieskiewicz A, Rutkowski R (2013) Transnasal endoscopic approaches to the cranial base. Polish Neurology and Neurosurgery.

31. Rappaport ZH (1993) Suprasellar arachnoid cyst: Options in operative management Acta Neurochir 122: 71-75.

32. Turhan T, Ersahin Y, Akinturk N et al (2012) Fenestration methods for Sylvian arachnoid cyst - endoscopy or microsurgery. Childs Nerv Syst 28: 229-235. [Crossref]

33. Oyama K, Fukuhara N, Taguchi M (2014) Transsphenoidal cyst cisternostomy with a keyhole dural opening for sellar arachnoid cyst: technical note. Neurosurg Rev.

34. Thompson TP, Lunsford L, Kondziolka D. (2000) Cyst with stereotactic intracavitary irradiation: An expanded report of four cases. Neurosurgery 46: 1518-1523.

35. Nowoslawska E, Mikolajczyk-Wieczorek W, Zakrzewski K (2009) Surgical treatment of hydrocephalus with the use neuroendoscopic techniques. Current Neurology 9: 1725 .

36. Mikolajczyk-Wieczorek W, Nowoslawska E (2009) Shunt system in the treatment of hydrocephalus. Current Neurology 9: 26-36.

37. Kapczuk I, Ben-Skowronek I, Jaklinska T (2010) Hormonal disorders in therapy of tumor of the hypothalamus and pituitary region. Pediatric Endocrinology. [Crossref]

Copyright: $₫ 2019$ Halabuda A. This is an open-access article distributed under the terms of the Creative Commons Attribution License, which permits unrestricted use, distribution, and reproduction in any medium, provided the original author and source are credited. 